\title{
Radiation from a Cavity-Backed Circular Aperture Surrounded by Concentric Corrugations
}

\author{
Ji Hyung Kim*, Sung Chan Song* and Yong Bae Park ${ }^{\dagger}$
}

\begin{abstract}
Radiation from a cavity-backed circular aperture surrounded by concentric corrugations is studied. An electromagnetic boundary-value problem of the cavity-backed circular aperture surrounded by concentric corrugations is solved by using the Green's function, Hankel transform, and mode matching method. The radiated fields are computed in terms of corrugation geometry to illustrate radiation behaviors and measured to validate our computation. The effects of the corrugation geometries on the radiation are discussed.
\end{abstract}

Keywords: Corrugation, Circular aperture, Cavity, Surface plasmon polarition

\section{Introduction}

Radiation from a cavity-backed circular aperture has been studied extensively due to its practical applications in aperture antennas and electromagnetic compatibility and aperture antennas [1-3]. Radiation from a cavitybacked single circular aperture has been analyzed by using the numerical techniques [1] and the mode matching technique [2]. Radiation from cavity-backed multiple circular apertures has also been investigated based on the mode matching technique [3]. Recently, there have been intensive studies on the extraordinary transmission through circular apertures surrounded by corrugations [4-6] due to the spoof surface plasmon polariton (SPP), which is bound electromagnetic surface waves mimicking the SPP in the optical region. It is well known that the corrugations on the output surface make the beamwidth of transmitted wave narrow by exciting the spoof SPP. These phenomena can be utilized for the aperture antenna with narrow beamwidth and a coaxially fed aperture antenna surrounded by corrugations has been proposed [7]. However, the theoretical analysis of cavity-backed circular aperture surrounded by concentric corrugations with an arbitrarily located feed within the cavity has not been presented. Unlike the coaxially fed structure with azimuthal symmetry in [7], arbitrary feed location without azimuthal symmetry need the full-wave analysis considering TM and TE modes.

In this paper, we the electromagnetic boundary value problem of cavity-backed circular aperture surrounded by concentric corrugations by using the Green's function, Hankel transform, and mode matching method. The radiated fields are represented in a series and computed in terms of corrugation geometry to illustrate radiation behaviors. A cavity-backed circular aperture surrounded

\footnotetext{
$\dagger$ Corresponding Author: Department of Electrical and Computer Engineering Ajou University. Korea. (yong@ajou.ac.kr) * Hanwha Systems, Korea. (\{jihyung.kim, sungchan.song\}@hanwha.com) Received: September 13, 2016; Accepted: December 8, 2016
}

by corrugations is fabricated and the radiation characteristic is measured to validate our computation. Also, the effects of the corrugation geometries on the radiation are discussed.

\section{Field Representations}

Assume that the z-oriented electric point source is located in a circular cavity with multiple circular apertures in a conducting plane, as shown in Fig 1. The time convention is suppressed throughout the analysis. In region (I), the total electric and magnetic fields are a sum of the incident and scattered components. The incident fields from the electric point source is represented based on the Green's function as [3]

$$
\begin{aligned}
E_{\rho}^{i}= & \frac{i}{\pi c^{2}} \sum_{n=1}^{\infty} \frac{e_{t m}^{I}}{\kappa_{0 n}^{(1)} \sin \kappa_{0 n}^{(1)}(h-d)\left[J_{0}{ }^{\prime}\left(\gamma_{0 n}^{(1)} c\right)\right]^{2}} \\
& \times\left\{\begin{array}{l}
\cos \kappa_{0 n}^{(1)}\left(z^{\prime}+d+t\right) \sin \kappa_{0 n}^{(1)}(z+d), \quad z^{\prime}<z<-d \\
\cos \kappa_{0 n}^{(1)}\left(z^{\prime}+d\right) \sin \kappa_{0 n}^{(1)}(z+d+t), \quad-d-t<z<z^{\prime}
\end{array}\right.
\end{aligned}
$$

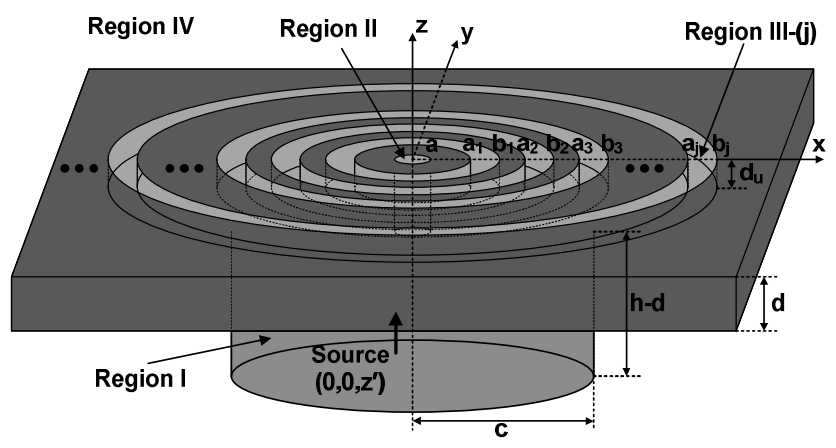

Fig. 1. Problem geometry 


$$
\begin{gathered}
H_{\varphi}^{i}=\frac{i}{\pi c^{2}} \sum_{n=1}^{\infty} \frac{h_{t m}^{I}}{\kappa_{0 n}^{(1)} \sin \kappa_{0 n}^{(1)}(h-d)\left[J_{0}{ }^{\prime}\left(\gamma_{0 n}^{(1)} c\right)\right]^{2}} \\
\times\left\{\begin{array}{l}
\cos \kappa_{0 n}^{(1)}\left(z^{\prime}+d+t\right) \sin \kappa_{0 n}^{(1)}(z+d), \quad z^{\prime}<z<-d \\
\cos \kappa_{0 n}^{(1)}\left(z^{\prime}+d\right) \sin \kappa_{0 n}^{(1)}(z+d+t), \quad-d-t<z<z^{\prime}
\end{array}\right. \\
E_{\rho}^{I}=i \sum_{n=1}^{\infty} A_{0 n} \sin \left[\kappa_{0 n}^{(1)}(z+h)\right] e_{t m}^{I} \\
H_{\phi}^{I}=\sum_{n=1}^{\infty} A_{0 n} \cos \left[\kappa_{0 n}^{(1)}(z+h)\right] h_{t m}^{I}
\end{gathered}
$$

where $k_{1}=\omega \sqrt{\mu_{1} \varepsilon_{1}}, \quad J_{0}\left(\gamma_{0 n}^{(1)} c\right)=0, \quad \kappa_{0 n}^{(1)}=\sqrt{k_{1}^{2}-\left(\gamma_{0 n}^{(1)}\right)^{2}}$, $e_{t m}^{I}=i \kappa_{0 n}^{(1)} \gamma_{0 n}^{(1)} J_{0}{ }^{\prime}\left(\gamma_{0 n}^{(1)} \rho\right)$, and $h_{t m}^{I}=i \omega \varepsilon_{1} \gamma_{0 n}^{(1)} J_{0}{ }^{\prime}\left(\gamma_{0 n}^{(1)} \rho\right)$.

In region (II) $(\rho<a,-d<z<0)$, at the aperture, the electric and magnetic fields are

$$
\begin{aligned}
& E_{\rho}^{I I}=\sum_{n=1}^{\infty}\left[B_{0 n} e^{i \kappa_{0 n}^{(2)} z}+C_{0 n} e^{-i \kappa_{0 n}^{(2)} z}\right] e_{t m}^{I I} \\
& H_{\phi}^{I I}=\sum_{n=1}^{\infty}\left[B_{0 n} e^{i \kappa_{0_{n}}^{(2)} z}-C_{0 n} e^{-i \kappa_{0 n}^{(2)} z}\right] h_{t m}^{I I}
\end{aligned}
$$

where $k_{2}=\omega \sqrt{\mu_{2} \varepsilon_{2}}, J_{0}\left(\gamma_{0 n}^{(2)} a\right)=0, \kappa_{0 n}^{(2)}=\sqrt{k_{2}^{2}-\left(\gamma_{0 n}^{(2)}\right)^{2}}$, $e_{t m}^{I I}=i \kappa_{0 n}^{(2)} \gamma_{0 n}^{(2)} J_{0}^{\prime}\left(\gamma_{0 n}^{(2)} \rho\right)$, and $h_{t m}^{I I}=i \omega \varepsilon_{2} \gamma_{0 n}^{(2)} J_{0}^{\prime}\left(\gamma_{0 n}^{(2)} \rho\right)$.

In region (III) $\left(a_{j}<\rho<b_{j},-d_{j}<z<0\right)$, at the $\mathrm{j}$-th corrugation, the electric and magnetic fields are

$$
\begin{aligned}
E_{\rho}^{I I I} & =i \sum_{n=1}^{\infty} D_{0 n}^{(j)} \sin \left[\kappa_{0 n}^{(3)(j)}\left(z+d_{j}\right)\right] e_{t m}^{I I I-(j)} \\
& +i V_{0}^{(j)} \sin \left[k_{3}^{(j)}\left(z+d_{j}\right)\right] e_{t e m}^{I I I-(j)} \\
H_{\phi}^{I I I} & =\sum_{n=1}^{\infty} D_{0 n}^{(j)} \cos \left[\kappa_{0 n}^{(3)(j)}\left(z+d_{j}\right)\right] h_{t m}^{I I I-(j)} \\
& +V_{0}^{(j)} \cos \left[k_{3}^{(j)}\left(z+d_{j}\right)\right] h_{t e m}^{I I I-(j)}
\end{aligned}
$$

where $k_{3}=\omega \sqrt{\mu_{3} \varepsilon_{3}}, \kappa_{0 n}^{(3)(j)}=\sqrt{k_{3}^{(j)^{2}}-\gamma_{0 n}^{(3)(j)^{2}}}, \Phi_{0}\left(\gamma_{0 n}^{(3)(j)} a_{j}\right)=0$, $\Phi_{0}\left(\gamma_{0 n}^{(3)(j)} \rho\right)=J_{0}\left(\gamma_{0 n}^{(3)(j)} a_{j}\right)-\frac{J_{0}\left(\gamma_{0 n}^{(3)(j)} b_{j}\right)}{N_{0}\left(\gamma_{0 n}^{(3)(j)} b_{j}\right)} N_{0}\left(\gamma_{0 n}^{(3)(j)} a_{j}\right)$, $e_{t e m}^{I I I}=\frac{1}{\rho}, \quad h_{t e m}^{I I I}=\sqrt{\frac{\varepsilon_{3}^{(j)}}{\mu}} \frac{1}{\rho}, \quad e_{t m}^{I I I}=i \kappa_{0 n}^{(3)(j)} \gamma_{0 n}^{(3)(j)} \Phi_{0}^{\prime}\left(\gamma_{0 n}^{(3)(j)} \rho\right)$, and $h_{t m}^{I I I}=i \omega \varepsilon_{3} \gamma_{0 n}^{(3)(j)} \Phi_{0}^{\prime}\left(\gamma_{0 n}^{(3)(j)} \rho\right)$.

In region (IV) $(z>0)$, the scattered electric and magnetic fields are represented based on the integral transform and superposition as [3]

$$
\begin{aligned}
& E_{\rho}^{I V}=\int_{0}^{\infty} \tilde{A}_{z}^{I V}(\zeta) e_{t m}^{I V} e^{i \kappa_{4} z} d \zeta \\
& H_{\phi}^{I V}=\int_{0}^{\infty} \tilde{A}_{z}^{I V}(\zeta) h_{t m}^{I V} e^{i \kappa_{4} z} d \zeta
\end{aligned}
$$

where $k_{4}=\omega \sqrt{\mu_{4} \varepsilon_{4}}, \quad \kappa_{4}=\sqrt{k_{4}^{2}-\zeta^{2}}, \quad e_{t m}^{I V}=i \kappa_{4} \zeta J_{0}^{\prime}(\zeta \rho)$, and $h_{t m}^{I V}=i \omega \varepsilon_{4} \zeta J_{0}^{\prime}(\zeta \rho)$.

\section{Enforcement of Boundary Conditions}

We enforce the boundary conditions to obtain a set of simultaneous equations for modal coefficients $A_{n_{n}}, B_{n_{n}}$, $C_{0 n}, D_{0 n}^{(j)}$ and $V_{0}^{(j)}(\mathrm{j}=1,2, ., \mathrm{N})$. Applying the power orthogonality to tangential fields at boundary $(z=-d, z=0)$ and carrying out algebraic manipulations gives a set of five simultaneous equations as

$$
\begin{aligned}
& E^{i}(\rho, \varphi,-d)+E^{I}(\rho, \varphi,-d) \\
& \quad=\left\{\begin{array}{cc}
E_{t}^{I I}\left(\rho_{t}, \varphi_{t},-d\right), & \rho_{t}<a_{t} \\
0, & \text { elsewhere }
\end{array}\right.
\end{aligned}
$$

Applying the power orthogonality to (11) and carrying out algebraic manipulations give

$$
\begin{aligned}
& i A_{0 q} \kappa_{0 q}^{(1)} \sin \left[\kappa_{0 q}^{(1)}(h-d)\right] \omega \varepsilon_{1} \frac{\gamma_{0 q}^{(1) 2} c^{2}}{2}\left[J_{0}{ }^{\prime}\left(\gamma_{0 q}^{(1)} c\right)\right]^{2} \\
& =\sum_{n=1}^{\infty}\left[B_{0 n} e^{-i \kappa_{0 n}^{(2)} d}+C_{0 n} e^{i \kappa_{0 n}^{(2)} d}\right] \\
& \quad \times \frac{\gamma_{0 q}^{(1)} J_{1}{ }^{\prime}\left(\gamma_{0 q}^{(1)} a\right) J_{1}\left(\gamma_{0 n}^{(2)} a\right)-\gamma_{0 n}^{(2)} J_{1}\left(\gamma_{0 q}^{(1)} a\right) J_{1}{ }^{\prime}\left(\gamma_{0 n}^{(2)} a\right)}{\gamma_{0 n}^{(2) 2}-\gamma_{0 q}^{(1) 2}} \\
& \quad \times \kappa_{0 n}^{(2)} \omega \varepsilon_{1} a
\end{aligned}
$$

Similarly, the continuity of tangential magnetic field at $z=-d$

$$
\begin{gathered}
H^{i}(\rho, \varphi,-d)+H^{I}(\rho, \varphi,-d) \\
=H_{t}^{I I}\left(\rho_{t}, \varphi_{t},-d\right), \rho<a .
\end{gathered}
$$

yields

$$
\begin{aligned}
& {\left[B_{0 q} e^{-i \kappa_{0 n}^{(2)} d}-C_{0 q} e^{i \kappa_{0 n}^{(2)} d}\right] \kappa_{0 q}^{(2)^{*}} \omega \varepsilon_{2} \frac{\gamma_{0 q}^{(2) 2} a^{2}}{2}\left[J_{0}{ }^{\prime}\left(\gamma_{0 q}^{(2)} a\right)\right]^{2}} \\
& =\sum_{n=1}^{\infty}\left[A_{0 n} \cos \left[\kappa_{0 n}^{(1)}(h-d)\right]\right. \\
& \left.\quad+\frac{1}{\pi c^{2}} \frac{\cos \left[\kappa_{0 n}^{(1)}\left(z^{\prime}+h\right)\right]}{\kappa_{0 n}^{(1)} \sin \left[\kappa_{0 n}^{(1)}(h-d)\right]\left[J_{0}{ }^{\prime}\left(\gamma_{0 n}^{(2)} c\right)\right]^{2}}\right] \\
& \times \frac{\gamma_{0 n}^{(1)} J_{1}{ }^{\prime}\left(\gamma_{0 n}^{(1)} a\right) J_{1}\left(\gamma_{0 q}^{(2)} a\right)-\gamma_{0 q}^{(2)} J_{1}\left(\gamma_{0 n}^{(1)} a\right) J_{1}{ }^{\prime}\left(\gamma_{0 q}^{(2)} a\right)}{\gamma_{0 q}^{(2) 2}-\gamma_{0 n}^{(1) 2}} \\
& \times \kappa_{0 q}^{(2) *} \omega \varepsilon_{1} a
\end{aligned}
$$


Tangential electric field continuities at $z=0$ require

$$
\begin{aligned}
& E^{I V}(\rho, \varphi, 0) \\
& =\left\{\begin{array}{cc}
E_{t}^{I I}(\rho, \varphi, 0), & \rho<a \\
E_{t}^{I I-(j)}\left(\rho_{j}, \varphi_{j}, 0\right), & a_{j}<\rho<b_{j} \\
0, & \text { elsewhere }
\end{array}\right.
\end{aligned}
$$

Applying the power orthogonality to (14) gives

$$
\begin{aligned}
\tilde{A}_{z}^{I V}(\zeta)= & \sum_{n=1}^{\infty}\left[B_{0 n}+C_{0 n}\right] \kappa_{0 n}^{(2)} \\
& \times \gamma_{0 n}^{(2)} a J_{0}{ }^{\prime}\left(\gamma_{0 n}^{(2)} a\right) \frac{\zeta J_{0}(\zeta a)}{\kappa_{4}\left(\zeta^{2}-\gamma_{0 n}^{(2) 2}\right)} \\
+ & \sum_{j=1}^{N} V_{0}^{(j)} \sin \left[k_{3}^{(j)} d_{j}\right]\left[J_{0}\left(\zeta b_{j}\right)-J_{0}\left(\zeta a_{j}\right)\right] \frac{1}{\kappa_{4} \zeta} \\
+ & \sum_{j=1}^{N} \sum_{n=1}^{\infty} D_{0}^{(j)} \sin \left[\kappa_{0 n}^{(3)(j)} d_{j}\right] \frac{2 \kappa_{0 n}^{(3)(j)}}{\pi} \\
& \times\left[\frac{J_{0}\left(\zeta a_{j}\right)}{N_{0}\left(\kappa_{0 n}^{(3)(j)} a_{j}\right)}-\frac{J_{0}\left(\zeta b_{j}\right)}{N_{0}\left(\kappa_{0 n}^{(3)(j)} b_{j}\right)}\right] \\
& \times \frac{\zeta}{\kappa_{4}\left(\zeta^{2}-\gamma_{0 n}^{(3)(j) 2}\right)}
\end{aligned}
$$

The continuity of tangential magnetic field at the aperture and $z=0$ is

$$
H^{I I}(\rho, \varphi, 0)=H_{t}^{I V}(\rho, \varphi, 0), \rho<a
$$

Substituting (16) into (17) and applying the power orthogonality to (17) and carrying out algebraic manipulations, we obtain

$$
\begin{aligned}
& {\left[B_{0 q}-C_{0 q}\right] \kappa_{0 q}^{(2)^{*}} \omega \varepsilon_{2} \frac{\gamma_{0 q}^{(2) 2} a^{2}}{2}\left[J_{0}{ }^{\prime}\left(\gamma_{0 q}^{(2)} a\right)\right]^{2} } \\
= & \sum_{n=1}^{\infty}\left[B_{0 n}-C_{0 n}\right] \kappa_{0 n}^{(2)} \omega \varepsilon_{4} \gamma_{0 n}^{(2)} a^{2} \\
& \times J_{0}{ }^{\prime}\left(\gamma_{0 n}^{(2)} a\right) \gamma_{0 q}^{(2)} J_{0}{ }^{\prime}\left(\gamma_{0 q}^{(2)} a\right) I_{3}^{a} \\
& +\sum_{j=1}^{N} V_{0}^{(j)} \sin \left[k_{3}^{(j)} d_{j}\right] \omega \varepsilon_{4} \gamma_{0 q}^{(2) 2} a I_{3}^{b} \\
& +\sum_{j=1}^{N} \sum_{n=1}^{\infty} D_{0 n}^{(j)} \sin \left[\kappa_{0 n}^{(3)(j)} d_{j}\right] \\
& \times \frac{2 \kappa_{0 n}^{(3)(j)} \omega \varepsilon_{4}}{\pi} \gamma_{0 q}^{(2)} a J_{0}{ }^{\prime}\left(\gamma_{0 q}^{(2)} a\right) I_{3}^{c}
\end{aligned}
$$

where

$$
I_{3}^{a}=\int_{0}^{\infty} \frac{\left[J_{0}(\zeta a)\right]^{2} \zeta^{3}}{\kappa_{4}\left(\zeta^{2}-\gamma_{0 n}^{(2) 2}\right)\left(\zeta^{2}-\gamma_{0 q}^{(2) 2}\right)} d \zeta
$$

$$
\begin{aligned}
& I_{3}^{b}=\int_{0}^{\infty}\left[J_{0}\left(\zeta b_{j}\right)-J_{0}\left(\zeta a_{j}\right)\right] \frac{J_{0}(\zeta a) \zeta}{\kappa_{4}\left(\zeta^{2}-\gamma_{0 q}^{(2) 2}\right)} d \zeta \\
& I_{3}^{c}=\int_{0}^{\infty}\left[\frac{J_{0}\left(\zeta a_{j}\right)}{N_{0}\left(\kappa_{0 n}^{(3)(j)} a_{j}\right)}-\frac{J_{0}\left(\zeta b_{j}\right)}{N_{0}\left(\kappa_{0 n}^{(3)(j)} b_{j}\right)}\right] \\
& \times \frac{\left[J_{0}(\zeta a)\right]^{2} \zeta^{3}}{\kappa_{4}\left(\zeta^{2}-\gamma_{0 n}^{(3)(j) 2}\right)\left(\zeta^{2}-\gamma_{0 q}^{(2) 2}\right)} d \zeta
\end{aligned}
$$

The continuity of tangential magnetic field at the $\mathrm{j}$-th corrugation and $z=0$ is

$$
H^{I I I-(j)}(\rho, \varphi, 0)=H_{t}^{I I}\left(\rho_{t}, \varphi_{t}, 0\right), a_{j}<\rho_{j}<b_{j}
$$

Applying the power orthogonality to (21) gives

$$
\begin{aligned}
& V_{0}^{(t)} \cos \left[k_{3}^{(t)} d_{t}\right] \sqrt{\frac{\varepsilon_{3}^{(t)}}{\mu_{0}}} \ln \frac{a_{t}}{b_{t}} \\
&=-i \sum_{n=1}^{\infty}\left[B_{0 n}+C_{0 n}\right] \kappa_{0 n}^{(2)} \omega \varepsilon_{4} \gamma_{0 n}^{(2)} a J_{0}{ }^{\prime}\left(\gamma_{0 n}^{(2)} a\right) I_{4}^{a} \\
&-i \sum_{j=1}^{N} V_{0}^{(j)} \sin \left[k_{3}^{(j)} d_{j}\right] \omega \varepsilon_{4} I_{4}^{b} \\
&+\sum_{j=1}^{N} \sum_{n=1}^{\infty} D_{0 n}^{(j)} \sin \left[\kappa_{0 n}^{(3)(j)} d_{j}\right] \frac{2 \kappa_{0 n}^{(3)(j)} \omega \varepsilon_{4}}{\pi} I_{4}^{c} \\
& D_{0 n}^{(t)} \cos \left[\kappa_{0 q}^{(3)(t)} d_{t}\right] \frac{2 \omega \varepsilon_{3}^{(t)}}{\pi} \\
& \quad+\left[\frac{1}{\left[N_{0}\left(\kappa_{0 q}^{(3)(t)} b_{t}\right)\right]^{2}}-\frac{1}{\left[N_{0}\left(\kappa_{0 q}^{(3)(t)} a_{t}\right)\right]^{2}}\right] \\
&=\sum_{n=1}^{\infty}\left[B_{0 n}+C_{0 n}\right] \kappa_{0 n}^{(2)} \frac{2 \omega \varepsilon_{4}}{\pi} \gamma_{0 n}^{(2)} a J_{0}{ }^{\prime}\left(\gamma_{0 n}^{(2)} a\right) I_{5}^{a} \\
&+\sum_{j=1}^{N} V_{0}^{(j)} \sin \left[k_{3}^{(j)} d_{j}\right] \frac{2 \omega \varepsilon_{4}}{\pi} I_{5}^{b} \\
&+\sum_{j=1}^{N} \sum_{n=1}^{\infty} D_{0 n}^{(j)} \sin \left[\kappa_{0 n}^{(3)(j)} d_{j}\right] \frac{4 \kappa_{0 n}^{(3)(j)} \omega \varepsilon_{4}}{\pi^{2}} I_{5}^{c}
\end{aligned}
$$

where

$$
\begin{gathered}
I_{4}^{a}=\int_{0}^{\infty}\left[J_{0}\left(\zeta b_{t}\right)-J_{0}\left(\zeta a_{t}\right)\right] \frac{J_{0}(\zeta a) \zeta}{\kappa_{4}\left(\zeta^{2}-\gamma_{0 n}^{(2) 2}\right)} d \zeta \\
I_{4}^{b}=\int_{0}^{\infty}\left[J_{0}\left(\zeta b_{j}\right)-J_{0}\left(\zeta a_{j}\right)\right]\left[J_{0}\left(\zeta b_{t}\right)-J_{0}\left(\zeta a_{t}\right)\right] \\
\times \kappa_{4} \zeta d \zeta \\
I_{4}^{c}=\int_{0}^{\infty}\left[\frac{J_{0}\left(\zeta a_{j}\right)}{N_{0}\left(\kappa_{0 n}^{(3)(j)} a_{j}\right)}-\frac{J_{0}\left(\zeta b_{j}\right)}{N_{0}\left(\kappa_{0 n}^{(3)(j)} b_{j}\right)}\right] \\
\times \frac{\left[J_{0}\left(\zeta b_{t}\right)-J_{0}\left(\zeta a_{t}\right)\right] \zeta}{\kappa_{4}\left(\zeta^{2}-\gamma_{0 n}^{(3)(j) 2}\right)} d \zeta
\end{gathered}
$$




$$
\begin{aligned}
& I_{5}^{a}=\int_{0}^{\infty}\left[\frac{J_{0}\left(\zeta a_{t}\right)}{N_{0}\left(\kappa_{0 n}^{(3)(t)} a_{t}\right)}-\frac{J_{0}\left(\zeta b_{t}\right)}{N_{0}\left(\kappa_{0 n}^{(3)(t)} b_{t}\right)}\right] \\
& \times \frac{J_{0}(\zeta a) \zeta^{3}}{\kappa_{4}\left(\zeta^{2}-\gamma_{0 n}^{(2) 2}\right)\left(\zeta^{2}-\gamma_{0 q}^{(3)(t) 2}\right)} d \zeta \\
& I_{5}^{b}= \int_{0}^{\infty}\left[\frac{J_{0}\left(\zeta a_{t}\right)}{N_{0}\left(\kappa_{0 n}^{(3)(t)} a_{t}\right)}-\frac{J_{0}\left(\zeta b_{t}\right)}{N_{0}\left(\kappa_{0 n}^{(3)(t)} b_{t}\right)}\right] \\
& I_{5}^{c}=\int_{0}^{\infty} {\left[\frac{\left[J_{0}\left(\zeta b_{j}\right)-J_{0}\left(\zeta a_{j}\right)\right] \zeta}{\kappa_{4}\left(\zeta^{2}-\gamma_{0 q}^{(3)(t) 2}\right)} d \zeta a_{t}\right) } \\
&\left.\times \frac{J_{0}\left(\zeta b_{t}\right)}{\left.N_{0 n}^{(3)(t)} a_{t}\right)}-\frac{\left.J_{0 n}^{(3)(t)} b_{t}\right)}{N_{0}\left(\zeta a_{j}\right)}-\frac{J_{0}\left(\zeta b_{j}\right)}{N_{0}\left(\kappa_{0 n}^{(3)(j)} b_{j}\right)}\right] \\
& \times \frac{\zeta_{0}\left(\kappa_{0 n}^{(3)(j)} a_{j}\right)}{\kappa_{4}\left(\zeta^{2}-\gamma_{0 n}^{(3)(j) 2}\right)\left(\zeta^{2}-\gamma_{0 q}^{(3)(t) 2}\right)} d \zeta .
\end{aligned}
$$

It is possible to solve a set of the simultaneous Eqs. (12), (14). (18), (23), and (24) for the modal coefficients $A_{0 n}$, $B_{0 n}, C_{0 n}, D_{0 n}^{(j)}$ and $V_{0}^{(j)}(\mathrm{j}=1,2, ., \mathrm{N})$.

\section{Convergence Check}

Before proceeding with the computation of radiated fields, it is worthwhile to investigate the convergence of modal coefficients in detail. Table 1 shows convergence behaviors of the modal coefficients in circular aperture and corrugations. Our extensive experience indicates that the modal orders to acquire numerical convergence should be those of propagating modes plus a few evanescent modes. Therefore, the numbers of modes used in computation are $\mathrm{Na}=6$ (radial direction) for the aperture (1 propagating

Table 1. The convergence behaviors of the modal coefficients (aperture, corrugations) of Fig. 3

\begin{tabular}{c|c|c|c|c|c}
\hline & $\mathrm{n}=1$ & $\mathrm{n}=2$ & $\mathrm{n}=3$ & $\mathrm{n}=4$ & $\mathrm{n}=5$ \\
\hline$\left|B_{0 n}\right|$ & $3.02 \mathrm{e}-4$ & $1.82 \mathrm{e}-7$ & $4.47 \mathrm{e}-9$ & $1.99 \mathrm{e}-10$ & $1.18 \mathrm{e}-11$ \\
\hline$\left|C_{0 n}\right|$ & $1.32 \mathrm{e}-4$ & $5.49 \mathrm{e}-6$ & $1.92 \mathrm{e}-6$ & $9.72 \mathrm{e}-7$ & $5.86 \mathrm{e}-7$ \\
\hline$\left|D_{0 n}^{(1)}\right|$ & $8.54 \mathrm{e}-8$ & $2.28 \mathrm{e}-12$ & $5.02 \mathrm{e}-13$ & $6.42 \mathrm{e}-15$ & $2.75 \mathrm{e}-18$ \\
\hline$\left|D_{0 n}^{(2)}\right|$ & $5.67 \mathrm{e}-8$ & $3.53 \mathrm{e}-10$ & $3.96 \mathrm{e}-13$ & $5.13 \mathrm{e}-15$ & $1.06 \mathrm{e}-18$ \\
\hline$\left|D_{0 n}^{(3)}\right|$ & $2.01 \mathrm{e}-8$ & $4.31 \mathrm{e}-13$ & $1.29 \mathrm{e}-13$ & $3.91 \mathrm{e}-15$ & $2.83 \mathrm{e}-18$ \\
\hline$\left|D_{0 n}^{(4)}\right|$ & $1.92 \mathrm{e}-8$ & $2.09 \mathrm{e}-10$ & $1.12 \mathrm{e}-13$ & $2.64 \mathrm{e}-15$ & $8.10 \mathrm{e}-18$ \\
\hline$\left|D_{0 n}^{(5)}\right|$ & $4.74 \mathrm{e}-8$ & $1.27 \mathrm{e}-13$ & $1.48 \mathrm{e}-13$ & $2.65 \mathrm{e}-15$ & $1.42 \mathrm{e}-18$ \\
\hline$\left|D_{0 n}^{(6)}\right|$ & $4.58 \mathrm{e}-8$ & $1.37 \mathrm{e}-10$ & $2.16 \mathrm{e}-13$ & $1.78 \mathrm{e}-15$ & $2.78 \mathrm{e}-18$ \\
\hline
\end{tabular}

mode and 5 evanescent modes) and corrugations (2 propagating modes and 5 evanescent modes) $\mathrm{Nc}=30$ for the cavity (15 propagating modes and 5 evanescent modes) to achieve convergence to within $0.01 \%$.

\section{Numerical Results}

Radiated fields from a cavity-backed circular aperture surrounded by concentric corrugations can be written by using stationary phase approximation as follow

$$
H_{\varphi}^{I V}=\tilde{A}_{z}^{I V}\left(k_{4} \sin \theta\right) i \omega \varepsilon_{4} k_{4} \cos \theta \frac{e^{i k_{4} r}}{r}
$$

In order to check the validity of our analysis, we fabricated circular aperture surrounded with concentric corrugations fed by coaxial line (See Fig. 2 (a) and (b)). Consider a circular cavity with circular aperture surrounded by 6 corrugations fed by coaxial line (See Fig. 2). The diameter of the flange of the fabricated circular cavity is $320 \mathrm{~mm}$. The coaxially fed monopole in a waveguide is approximately modeled as an electric line current [8]. The line current is assumed to be located in the cavity $\left(\mathrm{x}_{0}=0\right.$, $\left.\mathrm{y}_{0}=0, \mathrm{z}_{0}=-32 \mathrm{~mm}\right)$. Fig. 3 illustrates the radiation pattern versus the elevation angle $(\theta)$ at $15 \mathrm{GHz}$. The simulation results presented in this paper are carried out by using the MWS (time domain solver) of the CST Studio Suite 2015. Mesh size is $1 / 10 \lambda$ and convergence is $-30 \mathrm{~dB}$. The

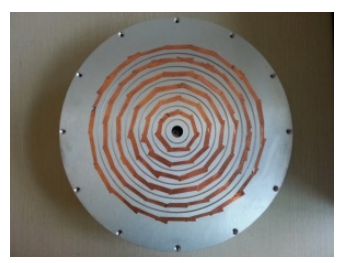

(a)

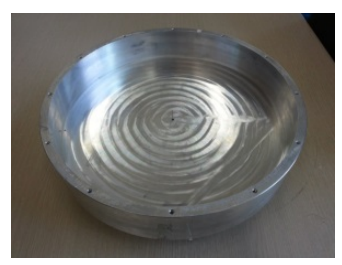

(b)
Fig. 2. (a) Fabricated circular cavity with circular aperture surrounded by corrugations antenna $(\mathrm{a}=8 \mathrm{~mm}, \mathrm{c}=$ $150 \mathrm{~mm}, \mathrm{du}=3 \mathrm{~mm}, \mathrm{~h}=47 \mathrm{~mm},\left(\mathrm{x}_{0}, \mathrm{y}_{0}, \mathrm{z}_{0}\right)=(0,0,-$ $32 \mathrm{~mm}), \mathrm{f}=15 \mathrm{GHz}$; (b) Coaxial feed

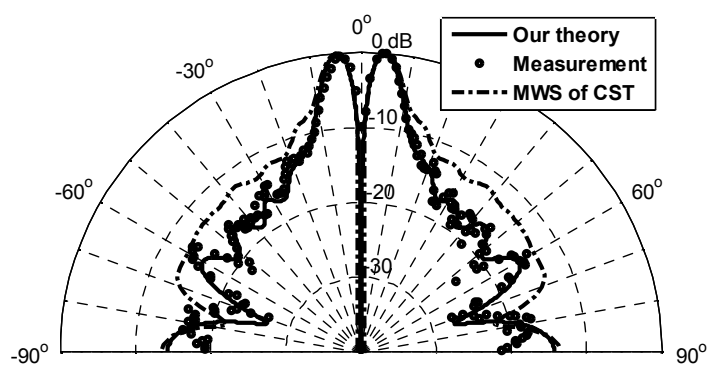

Fig. 3. Normalized radiation pattern versus elevation angle $(\theta)(\mathrm{a}=8 \mathrm{~mm}, \mathrm{c}=150 \mathrm{~mm}, \mathrm{~d}=5 \mathrm{~mm}, \mathrm{du}=3 \mathrm{~mm}, \mathrm{~h}$ $\left.=47 \mathrm{~mm},\left(\mathrm{x}_{0}, \mathrm{y}_{0}, \mathrm{z}_{0}\right)=(0,0,-32 \mathrm{~mm}), \mathrm{f}=15 \mathrm{GHz}\right)$ 
comparison between our results and measured data shows a good agreement. The discrepancies around 60 degree to 90 degree are due to the finite flange of the fabricated circular cavity and the measurement errors. It is seen that the simulation results do not show a good agreement with the computed and measured results. Fig. 3 is about 5 seconds whereas the MWS took about 2 hours and 50 minutes on a PC (Intel Core i7 3770, $3.4 \mathrm{GHz}, 8 \mathrm{~Gb}$ memory). Fig. 4 shows the radiation patterns for different

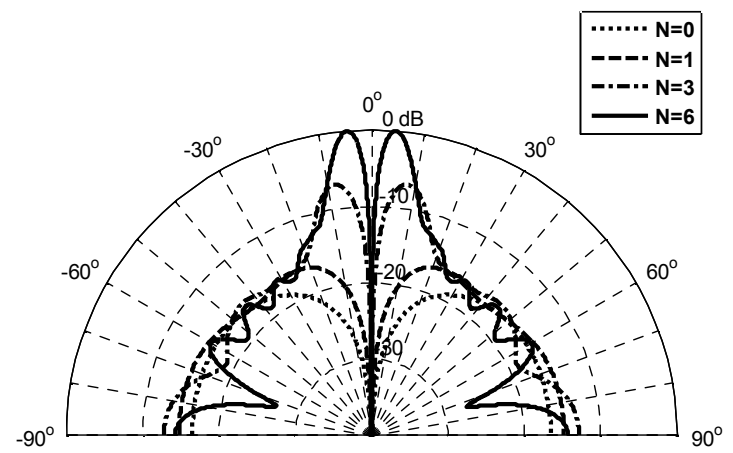

Fig. 4. Normalized radiation pattern versus elevation angle $(\theta)(\mathrm{a}=8 \mathrm{~mm}, \mathrm{c}=150 \mathrm{~mm}, \mathrm{~d}=5 \mathrm{~mm}, \mathrm{du}=3 \mathrm{~mm}, \mathrm{~h}$ $\left.=47 \mathrm{~mm},\left(\mathrm{x}_{0}, \mathrm{y}_{0}, \mathrm{z}_{0}\right)=(0,0,-32 \mathrm{~mm}), \mathrm{f}=15 \mathrm{GHz}\right)$.

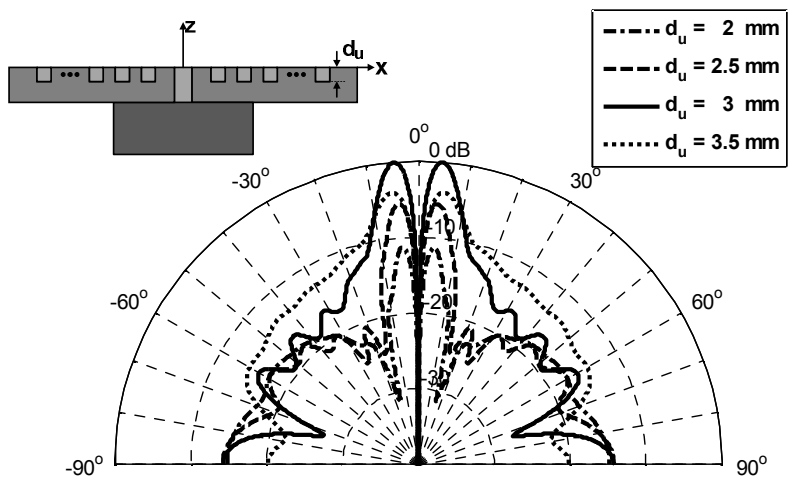

Fig. 5. Normalized radiation pattern versus elevation angle $(\theta)(\mathrm{a}=8 \mathrm{~mm}, \mathrm{c}=150 \mathrm{~mm}, \mathrm{~d}=5 \mathrm{~mm}, \mathrm{~h}=47 \mathrm{~mm}$, $\left.\left(\mathrm{x}_{0}, \mathrm{y}_{0}, \mathrm{z}_{0}\right)=(0,0,-32 \mathrm{~mm}), \mathrm{f}=15 \mathrm{GHz}\right)$

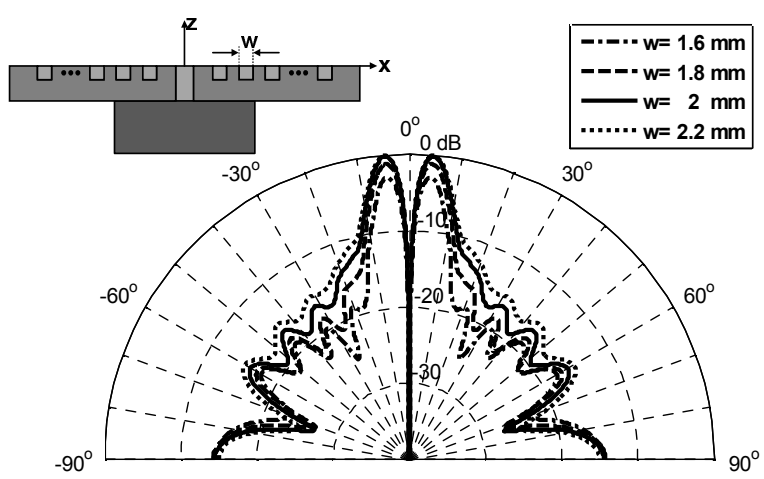

Fig. 6. Normalized radiation pattern versus elevation angle $(\theta)(\mathrm{a}=8 \mathrm{~mm}, \mathrm{c}=150 \mathrm{~mm}, \mathrm{~d}=5 \mathrm{~mm}, \mathrm{du}=3 \mathrm{~mm}, \mathrm{~h}$ $\left.=47 \mathrm{~mm},\left(\mathrm{x}_{0}, \mathrm{y}_{0}, \mathrm{z}_{0}\right)=(0,0,-32 \mathrm{~mm}), \mathrm{f}=15 \mathrm{GHz}\right)$ the number of corrugations. It is interesting to note that the main beam enhances as the number of aperture increases due to the excitations of SPP-like modes. Fig. 5 depicts the radiation pattern of cavity backed aperture surrounded by 6 corrugations for different depth of corrugations. Main beam has maximum value when $\mathrm{du}=3 \mathrm{~mm}$ due to the large excitation of SPP-like modes. In ideal cases, the optimum value of corrugation depth goes to $\lambda / 4$ when corrugation width is 0 . However, since we assumed corrugation width $2 \mathrm{~mm}$, it seems that the optimum value of corrugation depth decreases. Fig. 6. shows the radiation pattern of cavity backed aperture surrounded by 6 corrugations for different depth of corrugations width. Main beam power is saturated as width of corrugations increases. And side lobe gain increases. Therefore we choose $2 \mathrm{~mm}$ for optimum value of corrugation width.

\section{Conclusion}

The electromagnetic boundary-value problem of the cavity-backed circular aperture surrounded by concentric corrugations has been solved based on the Green's function, integral transform, superposition, and mode matching method. The radiated fields are computed in terms of corrugation geometry to illustrate radiation behaviors and measured to validate our computation. Our theoretical formulation is useful to design the cavity-backed aperture antenna with concentric corrugations.

\section{Acknowledgements}

This work was partly supported by the research fund of Signal Intelligence Research Center supervised by the Defense Acquisition Program Administration and the Agency for Defense Development of Korea and Basic Science Research Program through the National Research Foundation of Korea funded by the Ministry of Education (NO. 2014R1A1A2054467).

\section{References}

[1] C. J. Reddy, M. D. Deshpande, C. R. Cockrell, and F. B. Beck, "Radiation characteristics of cavity backed aperture antennas in finite ground plane using the hybrid FEM/MoM technique and geometrical theory of diffraction," IEEE Trans. Antennas Propag., vol. 44, no. 10, pp. 1327-1333, Oct. 1996.

[2] J. K. Paek and H. J. Eom, "Cylindrical cavity-backed antenna," IEEE Antennas Wireless Propag. Lett., vol. 8, pp. 852-855, Aug. 2009.

[3] J. H. Kim and Y. B. Park, "Electromagnetic Radiation from a Circular Cavity with Circular Apertures in a Conducting Plane," IEEE Trans. Antennas Propag., 
vol. 62, no. 9, pp. 4846-4951, Sep. 2014.

[4] D. Y. Na, J. H. Kim, K. Y. Jung, and Y. B. Park, "Extraordinary electromagnetic transmission through a circular aperture surrounded by surface corrugations," IET Microw. Antenna Propag., vol. 8, pp. 145157, Feb. 2014

[5] M. J. Lockyear, A. P. Hibbins, J. R. Sambles, and C. R. Lawrence, "Enhanced microwave transmission through a single subwavelength aperture surrounded by concentric grooves," J. Opt. A: Pure Appl., vol. 7, pp. s152-s158, 2005.

[6] H. Caglayan, I. Bulu, and E. Ozbay, "Extraordinary grating-coupled microwave transmission through a subwavelength annular aperture", Opt. Express, 13, (5), pp. 1666-1671, 2005.

[7] D. Y. Na, J. H. Kim, Y. B. Park, and K.-Y. Jung, "Mode Matching Analysis of a Coaxially Fed Annular Slot Surrounded with Corrugations," Electromagnetics, vol. 34, issue 2, pp. 92-110, Jan. 2014.

[8] J. M. Jarem, "A multifilament method-of-moments solution for the input impedance of a probe-excited semi-infinite waveguide," IEEE Trans. Microw. Theory Tech., vol. 35, no. 1, pp. 14.19, Jan. 1987.

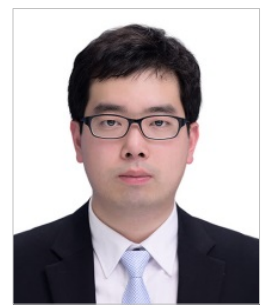

Jihyung Kim He obtained his B.S. and M.S./Ph.D. degree in electrical engineering from the Ajou University, Suwon, Korea, in 2009 and 2016, respectively. Since 2016, he has been a researcher at the Hanwha Systems. His research interests include analysis of aperture array antennas and radomes.

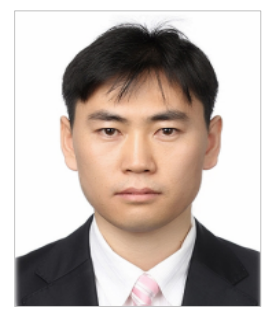

Sung Chan Song He obtained his B.S. and M.S. degree in avionics engineering from the Korea Aerospace University, Goyang, Korea, in 2001 and 2003, respectively. From 2002 to 2015, he was with Samsung Thales. Since 2015, he has been a researcher at the Hanwha systems. His research interests include antennas, electromagnetic wave numerical analysis, and radar system.

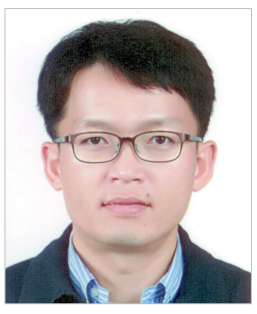

Yong Bae Park He received the B.S. (summa cum laude), M.S., and Ph.D. degrees in electrical engineering from Korea Advanced Institute of Science and Technology, Daejeon, Korea, in 1998, 2000, and 2003, respectively. From 2003 to 2006, he was a Researcher in Korea Telecom, Seoul, Korea. Since 2006, he has been with Ajou University, Suwon, Korea, where he is currently a Professor with the Department of Electrical and Computer Engineering. His current research interests include mode matching analysis of electromagnetic boundary-value problems, ray tracing analysis of radomes, and electromagnetic compatibility. Dr. Park received the Outstanding Researcher Award from Korean Institute of Electromagnetic Engineering Society in 2013. 